\title{
On Deciding Topological Classes of Deterministic Tree Languages
}

\author{
Filip Murlak * \\ Institute of Informatics, Warsaw University \\ ul. Banacha 2, 02-097 Warszawa, Poland \\ fmurlak@mimuw .edu.pl
}

\begin{abstract}
It has been proved by Niwiński and Walukiewicz that a deterministic tree language is either $\Pi_{1}^{1}$-complete or it is on the level $\Pi_{3}^{0}$ of the Borel hierarchy, and that it can be decided effectively which of the two takes place. In this paper we show how to decide if the language recognized by a given deterministic tree automaton is on the $\Pi_{2}^{0}$, the $\Sigma_{2}^{0}$, or the $\Sigma_{3}^{0}$ level. Together with the previous results it gives a procedure calculating the exact position of a deterministic tree language in the topological hierarchy.
\end{abstract}

Keywords: deterministic tree automata, index hierarchy, Borel hierarchy

\section{Introduction}

Tree automata, introduced by Rabin [14] in order to prove decidability of second order monadic logic of two successors, are today - together with $\mu$-calculus - the basic tool in modeling and verification of concurrent systems. A tree represents all possible behaviours of an analysed system and an automaton is a coded correctness condition. An interesting measure of complexity of such a condition is the nesting depth of positive and negative constraints on the events occurring infinitely often. The formalization of that criterion gives the notion of the index of an automaton. The languages recognized by automata of different indices constitute an ascending hierarchy. This hierarchy was proved to be strict for the classes of deterministic [19], nondeterministic [9], alternating [1, 7] and weak alternating automata [8].

Another approach to estimating the complexity of a language is to calculate its level in the topological hierarchy. Skurczyński [16] proved that the finite part of the Borel hierarchy is strict for languages recognized by weak alternating automata. Deterministic tree languages surprisingly turned out to be either $\Pi_{1}^{1}$ complete (hence non-Borel) or on the $\Pi_{3}^{0}$ level of the Borel hierarchy. The paper by Niwiński and Walukiewicz [11] containing the proof of the above suggests also that two basic complexity criteria, combinatorial and topological, are closely related - at least for deterministic automata.

\footnotetext{
* Supported by KBN Grant 4 T11C 04225.
} 
While the efficiency of verification methods depends on the brevity of the correctness conditions, they often result redundant when modeling real systems. Therefore, developing algorithmic methods for calculating actual automata's complexity would be interesting. So far, there have been presented procedures calculating the index of tree languages consisting of trees which have all the paths in a given regular $\omega$-language $[6,10]$, deciding if a deterministic automaton is equivalent to a Büchi automaton [18], and calculating the (nondeterministic) index of deterministic automata [12]. The $\mu$-calculus approach resulted in a procedure deciding if a given formula of modal $\mu$-calculus is equivalent to a formula of modal logic [13].

In this paper we concentrate on algorithmic calculation of a language's position in the topological hierarchy and its connections with the deterministic index hierarchy. In Sect. 2 and Sect. 3 we remind the basic notions of automata theory and present simple criteria determining a language's position in the deterministic index hierarchy and weak deterministic index hierarchy. Section 4 recalls some necessary topological notions. In Sect. 5 we show how to decide if a deterministic language is in the classes $\Sigma_{2}^{0}, \Pi_{2}^{0}$ and $\Sigma_{3}^{0}$. When combined with the previous characteristics by Niwiński and Walukiewicz, it provides a complete procedure calculating the position of a deterministic language in the topological hierarchy.

\section{Basic Notions}

We shall use the symbol $\omega$ to denote the set of natural numbers $\{0,1,2, \ldots\}$. For an alphabet $X, X^{*}$ is the set of finite words over $X$ and $X^{\omega}$ is the set of infinite words over $X$. The concatenation of words $u \in X^{*}$ and $v \in X^{*} \cup X^{\omega}$ will be denoted by $u v$, and the empty word by $\varepsilon$. The concatenation is naturally generalized for infinite sequences of words $v_{1} v_{2} v_{3} \ldots$. The concatenation of sets $A, B \subseteq X^{*}$ is $A B=\{u v: u \in A, v \in B\}$.

A binary tree is any subset of $\{0,1\}^{*}$ closed under the prefix relation. An element of a tree is usually called a node. A leaf is any node of a tree which is not a (strict) prefix of some other node. We shall be dealing mainly with labeled trees over $\Sigma$ which are functions $t: \operatorname{dom} t \rightarrow \Sigma$ such that dom $t$ is a tree. The symbol $T_{\Sigma}$ will denote the set of full infinite binary trees over $\Sigma$, i. e. functions $\{0,1\}^{*} \rightarrow \Sigma$.

For any trees $t, s$ and $v$, a node of $t$, the result of the substitution of $v$ with $s$ is a tree $t^{\prime}$ whose domain is the set $\operatorname{dom} t \cup v \operatorname{dom} s$ and

$$
t^{\prime}(u)=\left\{\begin{array}{l}
s\left(u^{\prime}\right) \text { if } u=v u^{\prime} \text { for some } u^{\prime} \\
t(u) \text { otherwise }
\end{array} .\right.
$$

Note that $u^{\prime}$ may be empty, so if $t(u) \neq s(\varepsilon)$, for the label of $u$ in $t^{\prime}$ we choose $s(\varepsilon)$. We find it more convenient since the state of an automaton in a node depends on every predecessor of the node, but not on the node itself.

The concatenation of tree languages $A, B$ is a tree language $A B$ consisting of all trees obtained from any $t \in A$ by substituting every leaf $u$ of $t$ with any tree $s_{u} \in B$. The concatenation of infinite sequence of tree languages is a natural 
generalization of the above. A more precise definition requires an auxiliary notion of a limit. Let $t_{0}, t_{1}, \ldots$ be a sequence of trees such that

- $\operatorname{dom} t_{0} \subseteq \operatorname{dom} t_{1} \subseteq \ldots$,

- $\forall v \in \bigcup_{m \in \omega} \operatorname{dom} t_{m} \quad \exists n_{v} \quad \forall n \geq n_{v} \quad t_{n}(v)=t_{n_{v}}(v)$.

The limit $t=\lim t_{n}$ is defined as follows:

$-\operatorname{dom} t=\bigcup_{m \in \omega} \operatorname{dom} t_{m}$,

$-t(v)=t_{n_{v}}(v)$.

An infinite concatenation of tree languages $L_{0} L_{1} \ldots$ consists of the limits of all sequences $t_{0}, t_{1}, \ldots$ such that $t_{0} \in L_{0}$ and $t_{n+1} \in\left\{t_{n}\right\} L_{n+1}$ for all $n$.

The concatenation of trees $s, t$ is the only element of the concatenation $\{s\}\{t\}$. Similarly, the concatenation of infinite sequence of trees $t=t_{1} t_{2} t_{3} \ldots$ is the only element of $\left\{t_{1}\right\}\left\{t_{2}\right\}\left\{t_{3}\right\} \ldots$

For $v \in \operatorname{dom} t$ we define $t . v$ as a subtree of $t$ rooted in $v$, i. e. $\operatorname{dom}(t \cdot v)=\{u$ : $v u \in \operatorname{dom} t\}, t \cdot v(u)=t(v u)$. A segment of a tree $t$ between $u$ and $u v$ is the restriction of the function t.u to the set $\{w \in \operatorname{dom}(t . u): v$ is not a strict prefix of $w\}$.

A (nondeterministic) automaton on words is a tuple $A=\left\langle\Sigma, Q, \delta, q_{0}\right.$, rank $\rangle$, where $\Sigma$ is a (finite) input alphabet, $Q$ is the set of states, $\delta \subseteq Q \times \Sigma \times Q$ is the relation of transition and $q_{0} \in Q$ is the initial state. The meaning of the function rank : $Q \rightarrow \omega$ will be explained later. Instead of $\left(q, \sigma, q_{1}\right) \in \delta$ one usually writes $q \stackrel{\sigma}{\longrightarrow} q_{1}$. A run of an automaton $A$ on a word $w \in \Sigma^{\omega}$ is a word $\rho_{w} \in Q^{\omega}$ such that $\rho_{w}(0)=q_{0}$ and if $\rho_{w}(n)=q, \rho_{w}(n+1)=q_{1}$, and $w(n)=\sigma$, then $q \stackrel{\sigma}{\longrightarrow} q_{1}$. A run $\rho_{w}$ is accepting if the highest rank repeating infinitely often in $\rho_{w}$ is even; otherwise $\rho_{w}$ is rejecting. A word is accepted by $A$ if there exist an accepting run on it. The language of words accepted by $A$ is denoted by $L(A)$. One says that $L$ is recognized by $A$ if $L=L(A)$. An automaton is deterministic if its relation of transition is a function $Q \times \Sigma \rightarrow Q$. Note, that we do not let the transition relation be a partial function, and so there is a run - accepting or not - on every word. We call a language deterministic if it is recognized by a deterministic automaton.

An (nondeterministic) automaton on trees is a tuple $A=\left\langle\Sigma, Q, \delta, q_{0}, \operatorname{rank}\right\rangle$, the only difference being that $\delta \subseteq Q \times \Sigma \times Q \times Q$. Like before, $q \stackrel{\sigma}{\longrightarrow} q_{1}, q_{2}$ means $\left(q, \sigma, q_{1}, q_{2}\right) \in \delta$. A run of $A$ on a tree $t \in T_{\Sigma}$ is a tree $\rho_{t} \in T_{Q}$ such that $\rho_{t}(\varepsilon)=q_{0}$ and if $\rho_{t}(v)=q, \rho_{t}(v 0)=q_{1}, \rho_{t}(v 1)=q_{2}$ and $t(v)=\sigma$, then $q \stackrel{\sigma}{\longrightarrow} q_{1}, q_{2}$. A path $\pi$ of the run $\rho_{t}$ is accepting if the highest rank repeating infinitely often in $\pi$ is even; otherwise $\pi$ is rejecting. A run is called accepting if all its paths are accepting. If at least one of them is rejecting, so is the whole run. An automaton is called deterministic if its transition relation is a function $Q \times \Sigma \rightarrow Q \times Q$.

An automaton is called weak if the rank of visited states does not increase during the run, i. e. whenever there is a transition $p \stackrel{\sigma}{\longrightarrow} q_{1}, q_{2}$, then $\operatorname{rank} p \geq$ $\operatorname{rank} q_{1}$ and $\operatorname{rank} p \geq \operatorname{rank} q_{2}$.

The symbol $G_{A}$ denotes a directed edge-labeled graph representing the transition relation of $A$. The set of vertices is $Q$ and whenever in $A$ there is a transition 
$p \stackrel{\sigma}{\longrightarrow} q_{1}, q_{2}$, in $G_{A}$ there is an edge between $p$ and $q_{1}$ labeled with $(\sigma, 0)$ and an edge between $p$ and $q_{2}$ labeled with $(\sigma, 1)$. For sake of brevity we shall write $p \stackrel{\sigma, 0}{\longrightarrow} q_{1}$ and $p \stackrel{\sigma, 1}{\longrightarrow} q_{2}$. A state is called productive if it is used in some accepting run. The productive graph $G_{A}^{+}$is analogous to $G_{A}$, only now the set of vertices is restricted to productive states and when defining the edges we demand that all of the states $p, q_{1}, q_{2}$ are productive. We shall call a path in $G_{A}$ productive if it is also a path in $G_{A}^{+}$.

A partial run of $A$ is a segment of any run of $A$. A partial run $\rho$ realizes a finite path $\pi$ in the graph $G_{A}^{+}$if it is a segment of an accepting run $\rho^{\prime}$ between two nodes $x$ and $y$ such that $\rho^{\prime}$ agrees with $\pi$ between $x$ and $y$. More precisely, if $\pi=p_{0} \stackrel{\sigma_{1}, d_{1}}{\longrightarrow} \ldots \stackrel{\sigma_{m}, d_{m}}{\longrightarrow} p_{m}$, then $y=x d_{1} d_{1} \ldots d_{m}, \rho^{\prime}(x)=p_{0}$ and $\rho^{\prime}\left(x d_{1} \ldots d_{i}\right)=$ $p_{i}$ for all $i$. Note that, since $\rho$ is a segment of an accepting run, all its infinite paths are accepting. A tree segment $f$ realizes a path $\pi$ if the corresponding partial run $\rho_{f}$ realizes $\pi$.

When analysing the way an automaton works, one finds it useful to let the automaton begin its run in states other than initial. An automaton starting in the state $q$ will be denoted by $A_{q}$.

The index of an automaton $A$ is a pair ( $\min \operatorname{rank} Q, \max \operatorname{rank} Q)$. Scaling down the rank function if necessary one may assume that $\min \operatorname{rank} Q \in\{0,1\}$. For an index $(i, j)$ we shall denote by $\overline{(i, j)}$ the dual index, i. e. $\overline{(0, j)}=(1, j+1)$, $\overline{(1, j)}=(0, j-1)$. The index hierarchy for a certain class of automata consists of (roughly speaking) ascending sets (levels) of languages recognized by $(i, j)$ automata, where $(i, j) \in\{0,1\} \times \omega$. It is known that index hierarchies are strict for deterministic [19], nondeterministic [9], alternating [1,7] and weak alternating automata [8].

\section{Deterministic Index Hierarchies}

Given a deterministic language, one may ask about its deterministic index, i. e. the exact position in the index hierarchy of deterministic automata. This question can be answered effectively. Here we follow the method introduced by Niwiński and Walukiewicz [10].

A sequence of loops $\lambda_{i}, \lambda_{i+1}, \ldots, \lambda_{j}$ in a graph of an automaton is called an alternating chain if the highest rank appearing on $\lambda_{k}$ has the same parity as $k$ and it is higher then the highest rank on $\lambda_{k-1}$. A $(i, j)$-flower is an alternating chain $\lambda_{i}, \lambda_{i+1}, \ldots, \lambda_{j}$ such that all loops start in the same state $q$. Let $\operatorname{Paths}(L) \subseteq \Sigma^{\omega}$ be the set of paths of trees from $L$ and $\operatorname{Paths}^{\prime}(L) \subseteq(\Sigma \times\{0,1\})^{\omega}$ denote the language of generalized paths of $L$,

$$
\operatorname{Paths}^{\prime}(L)=\left\{\left\langle\left(\sigma_{1}, d_{1}\right),\left(\sigma_{2}, d_{2}\right), \ldots\right\rangle: \exists t \in L t\left(d_{1} d_{2} \ldots d_{i-1}\right)=\sigma_{i}\right\}
$$

Niwiński and Walukiewicz show that a language $L$ is recognized by a $(i, j)$ automaton iff no deterministic automaton recognizing Paths $(L)$ contains a $\overline{(i, j)}$ flower. As an intermediate pass they prove the following fact. 
Theorem 1 (Niwiński, Walukiewicz [10]). A deterministic automaton on words is equivalent to a deterministic $(i, j)$-automaton iff it does not contain a $\overline{(i, j)}$-flower.

For a deterministic tree automaton $A$, the graph $G_{A}^{+}$can be treated as a deterministic automaton recognizing Paths' $(L(A))$. Conversely, given a deterministic word automaton recognizing Paths' $(L(A))$, one may interpret it as a graph of a tree automaton, obtaining thus a deterministic automaton recognizing $L(A)$. Hence, applying Theorem 1 one gets the following result.

Proposition 1. For a deterministic tree automaton $A$ the language $L(A)$ is recognized by a deterministic $(i, j)$-automaton iff $G_{A}^{+}$does not contain a $\overline{(i, j)}$ flower.

Similarly, one can calculate the exact position of a deterministic language in the hierarchy of weak deterministic automata. A weak $(i, j)$-flower is a sequence of loops $\lambda_{i}, \lambda_{i+1} \ldots, \lambda_{j}$ such that $\lambda_{k}$ is reachable in $G_{A}^{+}$from $\lambda_{k+1}$, and $\lambda_{k}$ is accepting iff $k$ is even. Intuitively, the notion is to provide long enough alternation of rank parity. Therefore we have to extend it to cover the case when $i$ is odd and instead of $\lambda_{i}$ there is an unproductive state $r$ reachable in $G_{A}$ from $\lambda_{i+1}$.

Proposition 2. For any deterministic tree automaton $A$ the language $L(A)$ can be recognized by a weak deterministic $(i, j)$-automaton iff $G_{A}^{+}$does not contain a weak $\overline{(i, j)}$-flower.

Proof. $(\Rightarrow)$ Let us suppose that $G_{A}^{+}$contains a weak $\left(i^{\prime}, j^{\prime}\right)$-flower, $\left(i^{\prime}, j^{\prime}\right)=\overline{(i, j)}$. Let $g_{j^{\prime}}$ be a tree segment realizing some path from the initial state $q_{0}$ to a state $r_{j^{\prime}}$ on $\lambda_{j^{\prime}}$. By induction, let $g_{k}$ realize a path from the state $r_{k+1} \in \lambda_{k+1}$ to a state $r_{k} \in \lambda_{k}$ for $k=j^{\prime}-1, \ldots, i^{\prime}$. Finally, let $f_{k}$ realize the loop $\lambda_{k}$ (from $r_{k}$ to $r_{k}$ ) for all $k$. Let $B$ be a weak deterministic automaton recognizing $L(A)$. Clearly, we can choose numbers $n_{i^{\prime}}, \ldots, n_{j^{\prime}}$ so that the run on $g_{j^{\prime}}\left(f_{j^{\prime}}\right)^{n_{j^{\prime}}} g_{j^{\prime}-1}\left(f_{j^{\prime}-1}\right)^{n_{j^{\prime}}-1} \ldots g_{i^{\prime}}\left(f_{i^{\prime}}\right)^{n_{i^{\prime}}}$ would need $j^{\prime}-i^{\prime}$ changes of rank parity and thus $j^{\prime}-i^{\prime}+1$ different ranks. Consequently, the index of $B$ cannot be $(i, j)$.

$(\Leftarrow)$ A weak deterministic $(i, j)$-automaton is obtained by setting $\operatorname{rank}(q)$ equal to the highest number $m$ such that there exists a weak $(\iota, m)$-flower with a path from $q$ to $\lambda_{m}$ (recall that an unproductive state is a weak $(1,1)$-flower).

Finally, for a deterministic language one may want to calculate its nondeterministic index, i.e. the position in the hierarchy of nondeterministic automata. This may be lower than the deterministic index, due to greater expressive power of nondeterministic automata. Consider for example the language $L_{M}^{\omega^{\omega}}$ consisting of trees whose leftmost paths are in a regular $\omega$-language $M$. It can be recognised by a (nondeterministic) Büchi automaton, but its deterministic index is equal to the deterministic index of $M$, which can be arbitrarily high.

The problem transpired to be rather difficult and has only just been solved in [12]. The analogous problem for nondeterministic languages remains open. 


\section{Topological Hierarchy}

We start with a short recollection of elementary notions of descriptive set theory. For further information see [5].

Let $2^{\omega}$ be the set of infinite binary sequences with a metric given by the formula

$$
d(u, v)= \begin{cases}\left.2^{-\min \{i \in \omega:} u_{i} \neq v_{i}\right\} & \text { iff } u \neq v \\ 0 & \text { iff } u=v\end{cases}
$$

and $T_{\Sigma}$ be the set of infinite binary trees over $\Sigma$ with a metric

$$
d(s, t)=\left\{\begin{array}{ll}
2^{-\min \left\{|x|: x \in\{0,1\}^{*}, s(x) \neq t(x)\right\}} & \text { iff } s \neq t \\
0 & \text { iff } s=t
\end{array} .\right.
$$

Both $2^{\omega}$ and $T_{\Sigma}$, with the topologies induced by the above metrics, are Polish spaces (complete metric spaces with countable dense subsets). In fact, both of them are homeomorphic to the Cantor discontinuum.

The class of Borel sets of a topological space $X$ is the closure of the class of open sets of $X$ by countable sums and complementation. Within this class one builds so called Borel hierarchy. The initial (finite) levels of it are defined as follows:

$\Sigma_{1}^{0}$ - open relations, i. e. open subsets of $X^{n}$ for some $n<\omega$,

$\Pi_{k}^{0}$ - complements of relations from $\Sigma_{k}^{0}$,

$\Sigma_{k+1}^{0}-$ countable sums of relations form $\Pi_{k}^{0}$.

For example, $\Pi_{1}^{0}$ are closed relations, $\Sigma_{2}^{0}$ are $F_{\sigma}$ relations, and $\Pi_{2}^{0}$ are $G_{\delta}$ relations.

Even more general classes of sets form the projective hierarchy. We will need only its lowest level:

$\Sigma_{1}^{1}$ - analytical sets, i. e. projections of Borel relations,

$\Pi_{1}^{1}$ - complements of relations from $\Sigma_{1}^{1}$.

Let $\varphi: X \rightarrow Y$ be a continuous map of topological spaces. One says that $\varphi$ reduces $A \subseteq X$ to $B \subseteq Y$, if $\forall x \in X x \in A \leftrightarrow \varphi(x) \in B$. Note that if $B$ is in a certain class of the above hierarchies, so is $A$. For any class $\mathcal{C}$ a set $B$ is $\mathcal{C}$-hard, if for any set $A \in \mathcal{C}$ there exists a reduction of $A$ to $B$. The topological hierarchy is strict for Polish spaces, so if a set is $\mathcal{C}$-hard, it cannot be in any lower class. If a $\mathcal{C}$-hard set $B$ is also an element of $\mathcal{C}$, then it is $\mathcal{C}$-complete.

For a deterministic automaton $A$ one may define a function $\varphi_{A}: T_{\Sigma} \rightarrow$ $T_{\text {im (rank) }}$ so that $\varphi_{A}(t)(v)=\operatorname{rank}\left(\rho_{t}(v)\right)$, where $\rho_{t}$ is the run of $A$ on $t$. Note that $\varphi_{A}$ is a continuous map that reduces $L(A)$ to the set $P$ of all trees satisfying $A$ 's parity condition.

We shall continue with a handful of examples which will turn out useful later.

Example 1. Consider the set $P_{(1,2)} \subseteq T_{\{1,2\}}$ consisting of trees having infinitely many 2 s on every path. For each $n<\omega$ let $G_{n}$ be the set of all trees that have at least one 2 below the level $n$ on every path. From König lemma it follows that each $G_{n}$ is open. Clearly, $P_{(1,2)}=\bigcap_{n \in \omega} G_{n}$ and so it is a $\Pi_{2}^{0}$ set. 
Example 2. Let $P_{(0,1)}^{\text {fin }} \subseteq T_{\{0,1\}}$ be the set of trees in which there are only finitely many 1s. For any $n<\omega$ a set $F_{n} \subseteq T_{\{0,1\}}$ consisting of trees having no 1 s below the level $n$ is closed. $P_{(0,1)}^{\text {fin }}=\bigcup_{n \in \omega} F_{n}$, hence $P_{(0,1)}^{\text {fin }} \in \Sigma_{2}^{0}$.

Example 3. Let $L_{a}^{0^{*} 1^{\omega}} \subseteq T_{\{a, b\}}$ be the set of trees which have an $a$ on every path from the set $0^{*} 1^{\omega}$. Suppose that it is a $\Sigma_{2}^{0}$ set. Let $L_{a}^{0^{*} 1^{\omega}}=\bigcup_{n \in \omega} F_{n}, F_{n}$ is closed for all $n$. We claim that for every $n$ there exists $m_{n}$ such that in every tree from $F_{n}$ the letter $a$ occurs on the path $0^{n} 1^{\omega}$ above the level $m_{n}$. If there was no such number, then we could find a sequence $t_{k}$ of trees having no letters $a$ on the path $0^{n} 1^{\omega}$ above the level $l_{k}$, where $l_{1}<l_{2}<l_{3}<\ldots$ As $T_{\{a, b\}}$ is compact, there exists a subsequence $t_{k_{i}}$ convergent in $F_{n}$. However the limit of $t_{k_{i}}$ cannot be in $F_{n}$ for it has no letter $a$ on the path $0^{n} 1^{\omega}$. Now, consider a tree $t$ with $a$ in nodes $0^{n} 1^{m_{n}+1}$ and $b$ in other nodes. Clearly, $t \in L_{a}^{0^{*} 1^{\omega}}$, but $t \notin \bigcup_{n \in \omega} F_{n}$. This way we have shown that $L_{a}^{0^{*} 1^{\omega}} \notin \Sigma_{2}^{0}$.

Example 4. In quite a similar way one proves that the set $Q=\left(0^{*} 1\right)^{*} 0^{\omega}$ is not in $\Pi_{2}^{0}$ (in fact, it is $\Sigma_{2}^{0}$-complete).

Example 5. Let $L_{Q}^{0^{*} 1^{\omega}}$ denote the language of trees such that the rightmost path from every node of the form $0^{*}$ belongs to the language $Q$ defined above. We shall see that it is $\Pi_{3}^{0}$-complete, and therefore it is not in $\Sigma_{3}^{0}$. Let us take any $M=\bigcup_{i<\omega} X_{i}$ with $X_{i}$ in $\Sigma_{2}^{0}$. Since $Q$ is $\Sigma_{2}^{0}$-complete, for each $i$ there exists $f_{i}$ reducing $X_{i}$ to $Q$. One easily defines a continuous reduction of $M$ to $L_{Q}^{0^{*} 1^{\omega}}$ assigning to each $t$ a tree having the word $f_{i}(t)$ on the path $0^{i} 1^{\omega}$ for all $i$, and 0s in all the other nodes.

\section{Deciding Levels of Topological Hierarchy}

The basic tool for investigating automata's properties is the technique of gadgets or difficult patterns in the graph of an automaton. In the topological context, the general recipe goes like this. For every class identify a gadget satisfying the following condition: if the gadget appears in an automaton $A$, then it provides a reduction of some difficult language to $L(A)$; otherwise $L(A)$ is in the class considered.

Wagner used this technique successfully in his solution of the general problem of continuous reductions between $\omega$-languages [19]. For infinite words, the Borel hierarchy collapses at the level $\Delta_{3}^{0}$ and below it is strict. The levels $\Pi_{1}^{0}$ and $\Sigma_{1}^{0}$ correspond to weak deterministic $(1,2)$ and $(0,1)$ automata; the class $\Delta_{2}^{0}$ consists of all weak deterministic automata; $\Pi_{2}^{0}$ and $\Sigma_{2}^{0}$ are exactly deterministic Büchi and co-Büchi languages. We shall see that the situation for trees is slightly different.

We start with the gap property for deterministic tree languages. An automaton $A$ admits a split if in $G_{A}^{+}$there are two loops $q_{0} \stackrel{\rho, 0}{\rightarrow} q_{1} \rightarrow \ldots \rightarrow q_{0}$ and $q_{0} \stackrel{\sigma, 1}{\rightarrow} q_{2} \rightarrow \ldots \rightarrow q_{0}$ such that the highest ranks occurring on them are of different parity and the higher is odd. 
Theorem 2 (Niwiński, Walukiewicz [11]). For a deterministic automaton $A, L(A)$ is on the level $\Pi_{3}^{0}$ of the Borel hierarchy iff $A$ does not admit split; otherwise $L(A)$ is $\Pi_{1}^{1}$-complete (hence non-Borel).

Owing to this result, it is enough to decide if a language is on the levels $\Sigma_{1}^{0}, \Pi_{1}^{0}$, $\Sigma_{2}^{0}, \Pi_{2}^{0}, \Sigma_{3}^{0}$ and use the split criterion to get complete information on its position in the topological hierarchy. Before dealing with this task we shall see that it does not get any easier, and so, not only there exist non-Borel tree languages but even the Borel hierarchy for trees is higher than for words.

Proposition 3. The Borel hierarchy for deterministic tree languages is strict below $\Pi_{3}^{0}$.

Proof. The language $L_{a}^{0^{*}}$ consisting of trees having an $a$ on the leftmost path is open, but obviously is not closed, $L_{a}^{0^{*}} \in \Sigma_{1}^{0} \backslash \Pi_{1}^{0}$. An example of a language from $\Pi_{1}^{0} \backslash \Sigma_{1}^{0}$ can be $\left\{t_{0}\right\}$, where $t_{0}(v)=0$ for every node $v$. The set $Q$ can be reduced to $P_{(0,1)}^{f i n}$ by a map $\{0,1\}^{\omega} \ni w \mapsto t_{w} \in T_{0,1}$ where $t_{w}$ is a tree whose leftmost path is $w$ and having 0 in all the other nodes. Hence $P_{(0,1)}^{f i n} \in \Sigma_{2}^{0} \backslash \Pi_{2}^{0}$. It can also be easily seen that $L_{a}^{0^{*} 1^{\omega}}$ is a $\Pi_{2}^{0}$ set and we have already proved that it is not a $\Sigma_{2}^{0}$. Finally, the language $L_{Q}^{0^{*} 1^{\omega}}$ has been shown not to be in the $\Sigma_{3}^{0}$ class, but clearly is in $\Pi_{3}^{0}$.

Having seen the strictness of our confined hierarchy, we shall continue with the characterization of its levels. The description of the open and the closed languages is probably well known, so we state it here, together with a short proof, just for the sake of completeness.

Proposition 4. For any deterministic tree automaton $A$

1. $L(A)$ is closed iff $A$ is equivalent to a weak deterministic $(1,2)$-automaton ${ }^{1}$,

2. $L(A)$ is open iff $A$ is equivalent to a weak deterministic $(0,1)$-automaton.

Proof. We will prove only (1). First, suppose that $A$ is not equivalent to a weak deterministic $(1,2)$-automaton. It follows from Proposition 2 that in $G_{A}^{+}$there must be an accepting loop $\lambda_{2}$ reachable from a rejecting loop $\lambda_{1}$. Let $g_{1}$ realize a path from $q_{0}$ to some $q_{1} \in \lambda_{1}, g_{2}$ realize a path form $q_{1}$ to some $q_{2} \in \lambda_{2}$, and $f_{1}, f_{2}$ realize loops $\lambda_{1}$ (from $q_{1}$ to $q_{1}$ ), $\lambda_{2}$ (from $q_{2}$ to $q_{2}$ ) respectively. Consider $t_{n}=g_{1}\left(f_{1}\right)^{n} g_{2}\left(f_{2}\right)^{\omega}$ and $t=g_{1}\left(f_{1}\right)^{\omega}$. Clearly, $t_{n} \in L(A)$ and $t_{n} \rightarrow t$ when $n \rightarrow \infty$, but $t \notin L(A)$. Hence $L(A)$ is not closed.

Now, if $L(A)$ is recognized by a weak deterministic automaton $B$, then it is the inverse image of a point under the continuous map $\varphi_{B}$ and so it is closed.

The combinatorial characterization of $\Pi_{2}^{0}$-languages transpires to be equally elegant.

\footnotetext{
${ }^{1}$ Recall that we do not let an automaton stop. If we did, there should be $(0,0)$ instead of $(1,2)$.
} 
Theorem 3. For a deterministic tree automaton $A$, the language $L(A)$ is on the level $\Pi_{2}^{0}$ of the Borel hierarchy iff $A$ is equivalent to a deterministic Büchi automaton.

Proof. $(\Rightarrow)$ Suppose that $L(A)$ is not recognized by a deterministic Büchi automaton. From Proposition 1 it follows that in $G_{A}^{+}$there exist an accepting loop $\lambda_{0}$ and a rejecting loop $\lambda_{1}$ forming a $(0,1)$-flower. The loops $\lambda_{0}$ and $\lambda_{1}$ cannot be equal, so there is a node $q$ lying on both loops, such that the next edges going out of $q$ in $\lambda_{0}$ and $\lambda_{1}$ have different labels. Let us assume first that the edges are labeled with different letters $a \neq b$ :

$$
\begin{aligned}
& \lambda_{0}: q \stackrel{a, d_{0}}{\longrightarrow} r \longrightarrow \ldots \longrightarrow q, \\
& \lambda_{1}: q \stackrel{b, d_{1}}{\longrightarrow} s \longrightarrow \ldots \longrightarrow q .
\end{aligned}
$$

Let $f_{0}, f_{1}$ be tree segments realizing the loops $\lambda_{0}$ and $\lambda_{1}$ respectively (both from $q$ to $q$ ). Note that $f_{0}$ and $f_{1}$ 's roots are labeled with different letters $a$ and $b$. Consider a map $\varphi: 2^{\omega} \rightarrow T_{\Sigma}$ defined by the formula

$$
\varphi\left(x_{0} x_{1} x_{2} \ldots\right)=f f_{x_{0}} f_{x_{1}} f_{x_{2}} \ldots,
$$

where $f$ is a tree segment realizing a path from $q_{0}$ to $q$. The map $\varphi$ is continuous, since $d\left(\varphi(x), \varphi\left(x^{\prime}\right)\right) \leq d\left(x, x^{\prime}\right)^{\left|\lambda_{0}\right|}$. Thus we have reduced $\left(0^{*} 1\right)^{*} 0^{\omega}$ to $L(A)$, which, by Example 4, implies that $L(A)$ is not a $\Pi_{2}^{0}$ language.

The second case is slightly more sophisticated. We have

$$
\begin{aligned}
& \lambda_{0}: q \stackrel{a, 0}{\longrightarrow} r \longrightarrow \ldots \longrightarrow q \\
& \lambda_{1}: q \stackrel{a, 1}{\longrightarrow} s \longrightarrow \ldots \longrightarrow q
\end{aligned}
$$

(or dual). Consider a path in the graph $G_{A}^{+}$along the edges of the loop $\lambda_{1}$ starting from $q$. We claim that it must reach a node $q^{\prime}$ such that there is an edge $e$ from $q^{\prime}$ to $q^{\prime \prime \prime}$ labeled with the same direction ( 0 or 1 ) as the edge in the loop but with a different letter, e.g.

$$
\begin{gathered}
\lambda_{1}: q \stackrel{a, 1}{\longrightarrow} s \longrightarrow \ldots \longrightarrow q^{\prime} \stackrel{b, 0}{\searrow^{c, 0}} q^{\prime \prime \prime} \longrightarrow \ldots \longrightarrow q \\
q^{\prime \prime \prime}
\end{gathered}
$$

Were there no such an edge, all the runs starting in $q$ would loop on $\lambda_{1}$ and $q$ would be unproductive. Let $\pi_{0}$ be the path $q \stackrel{a, 1}{\longrightarrow} s \longrightarrow \ldots \longrightarrow q^{\prime} \stackrel{e}{\longrightarrow} q^{\prime \prime \prime}$. The state $q^{\prime \prime \prime}$ is productive, so we can extend $\pi_{0}$ to an infinite accepting path $\pi$ in $G_{A}^{+}$. For $f_{0}$ we choose a tree segment realizing both $\lambda_{0}$ and $\pi$. This is possible owing to the fact, that $\lambda_{0}$ and $\pi$ start with edges labeled with the same letter and different directions. As before, $f_{1}$ can be any tree segment realizing $\lambda_{1}$. Now we can continue like in the previous case.

$(\Leftarrow) L(A)$ can be reduced to $P_{(1,2)}$, which is a $\Pi_{2}^{0}$-set. 
We shall now continue with describing the $\Sigma_{2}^{0}$ languages. Recall the language $L_{a}^{0^{*} 1^{\omega}} \subseteq T_{\{a, b\}}$ consisting of trees which have an $a$ on every path from the set $0^{*} 1^{\omega}$. Even though one may easily construct a deterministic $(0,1)$-automaton recognizing this language, it is not a $\Sigma_{2}^{0}$ set. Since a simple analog of the $\Pi_{2}^{0}$ case condition has shown insufficient, a more careful analysis of the automaton's graph is needed. We will say that a node $v \in G_{A}^{+}$is accessible with a split if in $G_{A}^{+}$there exist an accepting loop $u_{1} \stackrel{\sigma, d_{0}}{\longrightarrow} u_{2} \longrightarrow \ldots \longrightarrow u_{1}$ and a path $u_{1} \stackrel{\sigma, d_{1}}{\longrightarrow} u_{2}^{\prime} \longrightarrow \ldots \longrightarrow v$, where $d_{0} \neq d_{1}$. We will say that a loop or a flower is accessible with a split, meaning that it contains a node accessible with a split.

Theorem 4. For a deterministic tree automaton $A$, the language $L(A)$ is on the level $\Sigma_{2}^{0}$ of the Borel hierarchy iff $A$ is equivalent to a deterministic $(0,1)$ automaton and $G_{A}^{+}$does not contain a rejecting loop accessible with a split.

Proof. $(\Rightarrow)$ Let us suppose that $L(A)$ is a $\Sigma_{2}^{0}$ language. To prove the equivalence to a deterministic $(0,1)$-automaton follow the dual version of the method used in the previous theorem. There exist a rejecting loop $\lambda_{1}$ and an accepting loop $\lambda_{0}$ forming a $(1,2)$-flower. Find tree segments $f_{0}, f_{1}$ realizing $\lambda_{0}, \lambda_{1}$. Make sure they are different by finding an accepting path $\pi$ leaving $\lambda_{1}$. The map $\varphi$ defined in the previous proof reduces $\left(1^{*} 0\right)^{\omega}$ to $\operatorname{im} \varphi \cap L(A)$. Were $L(A)$ a $\Sigma_{2}^{0}$ set, so would $\left(1^{*} 0\right)^{\omega}$, which, by Example 4 , is not true. Let us now concentrate on the second part of the condition. Suppose that $G_{A}^{+}$does contain a rejecting loop $\lambda_{1}$ accessible with a split from an accepting loop $\lambda_{0}$ along a path $\pi$. For $n \in \omega$ let $\pi_{n}$ be an infinite accepting path having a prefix $\pi\left(\lambda_{1}\right)^{n}$ but no prefixes $\pi\left(\lambda_{1}\right)^{m}$ for $m>n$ (find an edge leaving the rejecting loop $\lambda_{1}$ just as it was done in the second case of the previous proof) and $\pi_{\omega}=\pi\left(\lambda_{1}\right)^{\omega}$. For each $\alpha \in \omega+1=\omega \cup\{\omega\}$ consider a tree segment $f_{\alpha}$ realizing both $\lambda_{0}$ and $\pi_{\alpha}$, this being possible since the first edges of both paths are labeled with the same letter $\sigma$ and different directions $d_{0}, d_{1}$. For any $x=\left(x_{1}, x_{2}, \ldots\right) \in(\omega+1)^{\omega}$ let $t_{x}=f f_{x_{1}} f_{x_{2}} \ldots$, where $f$ is a tree segment realizing a path from the initial state $q_{0}$ to $u_{1}$. We shall define a continuous map $\varphi: T_{\{a, b\}} \rightarrow T_{\Sigma}$. For $s \in T_{\{a, b\}}$ let $y_{i}=\min \left(\left\{|w|: w \in 0^{i} 1^{*}, s(w)=a\right\} \cup \omega\right)$. Let $z_{i}=\max \left(y_{i}-i, 0\right)$ if $y_{i}<\omega$ and $z_{i}=\omega$ if $y_{i}=\omega$. Let us now set $\varphi(s)=t_{z}$, where $z=\left(z_{0}, z_{1}, \ldots\right)$. The map $\varphi$ reduces $L_{a}^{0^{*} 1^{\omega}}$ to $L(A)$. However, we have already shown that $L_{a}^{0^{*} 1^{\omega}}$ is not a $\Sigma_{2}^{0}$ language. Hence $G_{A}^{+}$cannot contain a rejecting loop accessible with a split.

$(\Leftarrow)$ Investigating the proof of Theorem 1 one easily observes that the reduction is careful enough not to introduce any rejecting loops accessible with a split, provided there are no such loops in the original automaton. Therefore, we may assume that $A$ is a $(0,1)$-automaton such that $G_{A}^{+}$does not contain a rejecting loop accessible with a split. A state is called relevant if it has the highest rank on some productive loop. We may change the ranks of productive irrelevant states to 0 , and assume from now on that the odd states are either relevant or unproductive. We claim that the odd states occur only finitely many times on accepting runs of $A$. Suppose that an odd state $p$ occurs infinitely many times in an accepting run $\rho$. Then it appears in an infinite number of incomparable nodes $v_{0}, v_{1}, \ldots$ of $\rho$. Let $\pi_{i}$ be a path of $\rho$ going through the node $v_{i}$. Since 
$2^{\omega}$ is compact, we may assume, passing to a subsequence, that the sequence $\pi_{i}$ converges to a path $\pi$. As $v_{i}$ are incomparable, at most one of them, say $v_{i_{0}}$, may lie on $\pi$. Let us remove $\pi_{i_{0}}$ from the sequence $\pi_{i}$. Consider the node $w_{i}$ in which $p$ occurs for the first time on $\pi_{i}$ after leaving $\pi$ and let $\pi_{i}^{0}$ be the path from the last common node of $\pi$ and $\pi_{i}$ to $w_{i}$. Cutting the loops off if needed, we may assume that $\left|\pi_{i}^{0}\right| \leq|Q|$ for all $i \in \omega$. Subsequently, there exist a path $\pi^{0}$ repeating infinitely often in the sequence $\pi_{0}^{0}, \pi_{1}^{0}, \ldots$ Moreover, the path $\pi$ is accepting, so the starting node of $\pi^{0}$ must lay on an accepting productive loop. As $p$ is productive, the assumption implies that it is relevant and, being odd, lies on some productive rejecting loop. Hence, $G_{A}^{+}$contains a rejecting loop accessible with a split - a contradiction. This way we have shown that $\varphi_{A}$ reduces $L(A)$ to $P_{0,1}^{f i n}$, and so $L(A)$ is a $\Sigma_{2}^{0}$ language.

Let us now consider the class $\Sigma_{3}^{0}$. Every deterministic $\Sigma_{3}^{0}$ language is, due to Theorem 2, in the $\Delta_{3}^{0}=\Pi_{3}^{0} \cap \Sigma_{3}^{0}$ class. Below we present a combinatorial description of this class of languages.

Theorem 5. For a deterministic tree automaton $A, L(A)$ is a $\Sigma_{3}^{0}$ set iff $G_{A}^{+}$ does not contain a $(0,1)$-flower accessible with a split.

Proof. First let us suppose that $G_{A}^{+}$contains a $(0,1)$-flower accessible with a split. Following the method used in Theorem 3 one easily finds a map reducing the language $L_{Q}^{0^{*} 1^{\omega}}$ to $L(A)$. Subsequently, $L(A)$ is not a $\Sigma_{3}^{0}$ language.

Now, suppose that $G_{A}^{+}$does not contain a $(0,1)$-flower accessible with a split. We shall find a $\Sigma_{3}^{0}$ representation of the set $R \subseteq T_{Q}$ of accepting runs of $A$. The theorem will follow since the map $T_{\Sigma} \ni t \mapsto \rho_{t} \in T_{Q}$ is continuous. Let us consider, then, the set $\mathcal{X}$ of strongly connected components of $G_{A}^{+}$. Recall that they form a directed acyclic graph, i. e. no path returns to a component it has left. The language $R$ can be expressed by the following formula

$$
R=\bigcap_{X \in \mathcal{X}} R_{X},
$$

where $R_{X}$ is the set of runs whose every path staying forever in $X$ is accepting. Owing to this simple observation, it is enough to prove that the sets $R_{X}$ are $\Sigma_{3}^{0}$.

Let $\Pi_{X}$ denote the set of all paths from the initial state $q_{0}$ to some state in $X$ containing only one state from $X$. Note that $\Pi_{X}$ is countable for every $X$. Let us first suppose that $X$ is accessible with a split. For $\pi \in \Pi_{X}$ let $R_{X, \pi}$ denote the set of runs whose every path going along $\pi$ either leaves $X$ or is accepting. By the hypothesis, $X$ contains no $(0,1)$-flowers, and so it can be replaced by an equivalent component $X^{\prime}$ using only ranks 1 and 2 . Therefore, given $q \in X^{\prime}$, the set of runs of $A_{q}$, whose all paths are accepting or leave $X^{\prime}$, is a $\Pi_{2}^{0}$ set. Obviously, so is $R_{X, \pi}$. As it also holds that

$$
R_{X}=\bigcap_{\pi \in \Pi_{X}} R_{X, \pi},
$$

$R_{X}$ is a $\Pi_{2}^{0}$ set. 
The case of $X$ not accessible with a split is slightly fastidious. Let $\rho$ be an accepting run of $A$. Consider $\rho_{X}$, a subtree of $\rho$ formed by the nodes which have a successor whose labeling state is in $X$. No state from $X$ is accessible with a split, therefore it cannot appear in infinitely many incomparable nodes of $\rho$. Hence, $\rho_{X}$ has only finitely many branches. Let $\rho_{X}^{0}$ denote the tree $\rho_{X}$ restricted to the highest level below which there are no branching points. Let $R_{X}^{0}$ denote the set of all such trees; note that, although $R_{X}$ may be uncountable, $R_{X}^{0}$ is countable. Obviously,

$$
R_{X}=\bigcup_{s \in R_{X}^{0}} R_{X, s}
$$

where $R_{X, s}$ is the set of runs from $R_{X}$ coinciding with a tree $s \in R_{X}^{0}$ in its domain. Observe that $R_{X, s}$ is equal to the set of runs $\rho^{\prime}$ satisfying the following conditions:

(1) $\rho^{\prime}$ coincides with $s$ in its domain,

(2) the states from $X$ appear only in successors of leaves of $s$,

(3) in every subtree of $\rho^{\prime}$ rooted in a leaf of $s$ the states from $X$ appear infinitely often on at most one path,

(4) in every subtree of $\rho^{\prime}$ rooted in a leaf of $s$ the highest rank of the states from $X$ appearing infinitely often is even.

The condition (1) obviously defines an open set. The condition (2) defines a closed set and so does the condition (3), because it is equivalent to saying that no node of the subtree has both children in $X$. The condition (4) is of the $B\left(\Sigma_{2}^{0}\right)$ type. By $B\left(\Sigma_{2}^{0}\right)$ we mean the closure of $\Sigma_{2}^{0}$ by the finite Boolean operations; it is clearly a subclass of $\Sigma_{3}^{0}$. Hence $R_{X, s}$ is a $\Sigma_{3}^{0}$ set and so is $R_{X}$.

As a conclusion we obtain the main result of this paper.

Corollary 1. The problem of calculating the exact position in the topological hierarchy of a language recognized by a deterministic tree automaton is decidable within the time of finding the productive states of a deterministic automaton.

Proof. From Proposition 4 it follows that the language recognised by a deterministic automaton $A$ is closed iff $A$ is equivalent to a weak $(1,2)$-automaton. This, by Proposition 2, can be reformulated as follows: $L(A)$ is closed iff $G_{A}^{+}$does not contain a weak $(0,1)$-flower. Now, to decide whether a deterministic automaton recognises a closed set, first determine its productive states, then build its productive graph and check for weak $(0,1)$-flowers. Note that two last steps can be easily done in polynomial time. The case of open languages is entirely dual.

For $\Pi_{2}^{0}$ and $\Sigma_{2}^{0}$ levels follow analogous argument only now using Theorem 3 and Theorem 4 respectively, and Proposition 1 . For $\Pi_{3}^{0}$ and $\Sigma_{3}^{0}$ levels use the gap property and Theorem 5 .

This way for a given deterministic language one obtains its exact level in the topological hierarchy. 
In general, deciding the topological complexity of a deterministic tree language is as difficult as calculating the unproductive states of an automaton, the latter being equivalent to deciding a language's emptiness. In 1969 Rabin [14] showed that the emptiness problem is decidable, and in 1988 Emerson and Jutla [3] presented an algorithm with time complexity $\mathcal{O}\left((n d)^{3 d}\right)$, where $n$ is the number of states and $d$ is the number of ranks used. The emptiness problem can be easily reduced to solving parity games. The late nineties brought improved algorithms for this problem by Browne et al. [2] with complexity $\mathcal{O}\left(d^{2} m n^{\frac{d}{2}}\right)$ and by Seidl [15] with complexity $\mathcal{O}\left(d m\left(\frac{n+d}{d}\right)^{\frac{d}{2}}\right)$, where $n, m$, and $d$ are the numbers of vertices, edges, and ranks in the game graph. The investigation of parity games resulted in polynomial algorithms in plenty of special cases, but so far it is not known if the original problem is polynomial. One of the last achievements in this field is the procedure by Jurdziński and Vöge [4] which is apparently quite efficient, however its complexity has not, at present, got any nontrivial upper bounds.

\section{Acknowledgment}

The author would like to thank Damian Niwiński for helpful comments and discussions, and the anonymous referees for helpful comments.

\section{References}

1. Bradfield, J. C.: The modal mu-calculus alternation hierarchy is strict. Theoret. Comput. Sci. 195 (1998) 133-153

2. Browne, A., Clarke, E. M., Jha, S., Long, D. E., Marrero, W.: An improved algorithm for the evaluation of fixpoint expressions. Theoret. Comput. Sci. 178 (1997) 237-255

3. Emerson, E. A., Jutla, C. S.: The complexity of tree automata and logics of programs. In: Proc. FoCS '88. IEEE Computer Society Press (1988) 328-337

4. Jurdziński, M., Vöge, J.: A discrete strategy improvement algorithm for solving parity games. In: Proc. CAV 2000. Lecture Notes in Computer Science, Vol. 1855. Springer-Verlag (2000) 202-215

5. Kechris, A. S.: Classical Descriptive Set Theory. Graduate Texts in Mathematics, Vol. 156. Springer-Verlag (1995)

6. Kupferman, O., Safra, S., Vardi, M.: Relating Word and Tree Automata. In: 11th IEEE Symp. on Logic in Comput. Sci. (1996) 322-332

7. Lenzi, G.: A hierarchy theorem for the mu-calculus. In: auf der Heide, F. M., Monien, B. (eds.): Proc. ICALP '96. Lecture Notes in Computer Science, Vol. 1099. SpringerVerlag (1996) 87-109

8. Mostowski, A. W.: Hierarchies of weak automata and weak monadic formulas. Theoret. Comput. Sci. 83 (1991) 323-335.

9. Niwiński, D.: On fixed point clones. In: Kott, L. (ed.): 13th ICALP '86. Lecture Notes in Computer Science, Vol. 226. Springer-Verlag (1986) 464-473

10. Niwiński, D., Walukiewicz, I.: Relating hierarchies of word and tree automata. In: STACS '98. Lecture Notes in Computer Science, Vol. 1373. Springer-Verlag (1998) $320-331$ 
11. Niwiński, D., Walukiewicz, I.: A gap property of deterministic tree languages. Theoret. Comput. Sci. 303 (2003) 215-231

12. Niwiński, D., Walukiewicz, I.: Deciding nondeterministic hierarchy of deterministic tree automata. In: Proc. WoLLiC 2004 (to appear in Electronic Notes in Theoretical Computer Science)

13. Otto, M.: Eliminating recursion in $\mu$-calculus. In: STACS'99. Lecture Notes in Computer Science, Vol. 1563. Springer-Verlag (1999) 531-540

14. Rabin, M. O.: Decidability of second-order theories and automata on infinite trees. Trans. Amer. Soc. 141 (1969) 1-35

15. Seidl, H.: Fast and simple nested fixpoints. Information Processing Letters 59 (1996) 303-308

16. Skurczyński, J.: The Borel hierarchy is infinite in the class of regular sets of trees. Theoret. Comput. Sci. 112 (1993) 413-418

17. Thomas, W.: Languages, automata, and logic. In: Rozenberg, G., Salomaa, A. (eds.): Handbook of Formal Languages, Vol. 3. Springer-Verlag (1997) 389-455

18. Urbański, T. F.: On deciding if deterministic Rabin language is in Büchi class. In: Montanari, J. R. U., Welzl, E. (eds.): Proc. ICALP 2000. Lecture Notes in Computer Science, Vol. 1853. Springer-Verlag (2000) 663-674

19. Wagner, K.: Eine topologische Charakterisierung einiger Klassen regulärer Folgenmengen. J. Inf. Process. Cybern. EIK 13 (1977) 473-487 